\title{
VALUE OF DIFFUSION-WEIGHTED MRI IN BIRADS 3 AND 4 BREAST LESIONS
}

\author{
Lekshmy R1, Sandya C. J2, Srikanth Moorthy33, Resmi Sekhar4, Sreekumar K. P5, Rajesh Kannan6 \\ ${ }^{1}$ Senior Resident, Department of Radiology, Amrita Institute of Medical Sciences, Cochin. \\ ${ }^{2}$ Associate Professor, Department of Radiology, Amrita Institute of Medical Sciences, Cochin. \\ ${ }^{3}$ Clinical Professor and HOD, Department of Radiology, Amrita Institute of Medical Sciences, Cochin. \\ ${ }^{4}$ Assistant Professor, Department of Radiology, Amrita Institute of Medical Sciences, Cochin. \\ 5 Professor, Department of Radiology, Amrita Institute of Medical Sciences, Cochin. \\ ${ }^{6}$ Associate Professor, Department of Radiology, Amrita Institute of Medical Sciences, Cochin.
}

ABSTRACT

\section{BACKGROUND}

Breast cancer is the second leading cause of cancer deaths in women today. Important risk factors for female breast cancer include age (increases as women gets older), genetics, early onset of menarche and late age of menopause, family or personal history of breast cancer, obesity, nulliparity, use of HRT and OCPs, consumption of alcohol and a middle to high socioeconomic status. Diffusionweighted imaging is a new MR imaging technique in which contrast within the image is based on microscopic motion of water. It is reported to be highly sensitive for evaluation of breast cancer and a possible means of differentiating benign and malignant tumour. Diffusion is quantified by measuring apparent diffusion coefficient (ADC). This study was performed to determine whether diffusionweighted imaging can be useful for diagnosis of mammographically detected BIRADS 3 \& 4 lesions, correlating with histopathological diagnosis and to derive ADC values which may allow distinguishing benign from malignant lesions.

\section{MATERIALS AND METHODS}

The cross sectional study included 50 women with suspicious breast lesion on mammogram who underwent DWI before biopsy. Morphology, kinetic features, diffusion-weighted appearance of lesions and ADCs were evaluated. Apparent diffusion co-efficient values were calculated from DWI and compared for benign and malignant lesions and correlated with histopathology in all cases.

\section{RESULTS}

In this study, $\mathrm{ADC}$ cut-off for benign and malignant lesions was set as $1.3 \times 10(-3)$ square mm/sec. for ADC provided $80.8 \%$ sensitivity and $100 \%$ specificity. ADC in characterising benign and malignant lesions was significant with p- value of 0.004 (applying $\mathrm{t}$-test). Diffusion-weighted appearances of benign and malignant lesion turned out to be statistically significant (McNemar's test, $p$ value0.18). Study also proved that BIRADS 4 categorisation on its own has a sensitivity of $84.62 \%$ and specificity of $75 \%$.

\section{CONCLUSION}

This study showed that ADC is an effective and precise parameter to identify and distinguish between malignant and benign breast lesions. The corresponding ADC values which can be used to distinguish benign from malignant are also derived as a part of this study. Diffusion-weighted imaging is found to be useful in diagnosis of BIRADS 3 \& 4 lesions.

\section{KEYWORDS}

Diffusion MRI, BIRADS 3, BIRADS 4, Mammography.

HOW TO CITE THIS ARTICLE: Lekshmy R, Sandya CJ, Moorthy S, et al. Value of diffusion-weighted MRI in BIRADS 3 and 4 breast lesions. J. Evolution Med. Dent. Sci. 2017;6(31):2557-2562, DOI: 10.14260/Jemds/2017/553

\section{BACKGROUND}

Carcinoma breast is a significant cause of worldwide cancer morbidity and mortality. Breast cancer is the second leading cause of cancer deaths in women today.(1) Important risk factors for female breast cancer include age (increases as women gets older), genetics (mutation of BRCA 1 \& 2 genes), early onset of menarche and late age of menopause, family history of breast cancer in mother or sister, a personal history of breast cancer, obesity, nulliparity, use of HRT and OCPs,

Financial or Other, Competing Interest: None.

Submission 21-11-2016, Peer Review 10-01-2017,

Acceptance 16-01-2017, Published 17-04-2017.

Corresponding Author:

Dr. Lekshmy R,

Devamritam, \#19, Shantinagar Lane,

Chakkarapadambu,

Vytilla, Cochin-682028.

E-mail: lekshmyskandan@gmail.com

DOI: $10.14260 /$ jemds $/ 2017 / 553$

\section{(c) $(1) \odot$}

consumption of alcohol and a middle to high socioeconomic status. Triple assessment is essential to evaluate breast lesions, which include self-examination, imaging and histological diagnosis.

Diffusion-weighted imaging is a new MR imaging technique in which contrast within the image is based on microscopic motion of water. It is reported to be highly sensitive for evaluation of breast cancer and a possible means of differentiating benign and malignant tumour. Diffusion is quantified by measuring apparent diffusion coefficient (ADC).(2)

This study was performed to determine whether DWI can be useful for diagnosis of mammographically detected BIRADS 3 \& 4 lesions, correlating with histological diagnosis and to derive ADC values which may allow to distinguish benign from malignant breast lesions. 
MATERIALS AND METHODS

Study Period

August 2011 - September 2013.

\section{Inclusion Criteria}

Patients who are found to be in BIRADS - $3 \& 4$ category on mammography.

\section{Study Design}

Cross-sectional study.

\section{Sample Size}

Based on the results on sensitivity and specificity of diffusionweighted imaging and conventional MR mammogram appearance of the lesion with histopathology from the available literature [Reference: Contribution of DiffusionWeighted Imaging to Dynamic Contrast-Enhanced MRI in the characterisation of Breast tumours: AJR2011; 196; 210217)] ${ }^{(3)}$ and with $99 \%$ confidence and $10 \%$ allowable error minimum sample size comes to 10 . This study included fifty cases.

\section{Methods}

\section{Imaging Techniques}

1. Mammogram is done using Siemens 3000 Nova Mammomat Mammography unit. X-rays are produced in range of $24-32 \mathrm{KVp}$ with $\mathrm{mA}$ values ranging from 100 150. Craniocaudal \& mediolateral oblique views are taken usually. Spot compression view, magnification view, true lateral view, are done in areas that are suspicious on routine mammogram.

2. MRI Breast is done using GE SIGNA HDXT 1.5T MRI unit with dedicated HD 8 channel Vibrant breast array coil. Patient is placed in prone position on a dedicated breast coil.

Axial and Sagittal T1 and T2 images are obtained. T1 weighted images are acquired using a TR of $480-500 \mathrm{~ms}$ and TE of $10 \mathrm{~ms}$. T2 weighted images are acquired using TR of $3000 \mathrm{~ms} \& \mathrm{TE}$ of $100 \mathrm{~ms}$.

3. Axial DWI with single shot echoplanar imaging is performed at $b$ values 0 and $700 \mathrm{~mm} / \mathrm{s}$. Diffusionweighted images are obtained by adding a series of two sequential gradient pulses to a 90 - 180 degrees spinecho sequence. The first gradient pulse is applied between the 90 and the 180-degree pulse. Motion after this pulse causes molecules to acquire phase shifts of their transverse magnetisation. The second gradient pulse is applied immediately after 180 degrees.

ADC values are automatically calculated by placing ROI (Region of Interest) well within the confines of the lesion. Fatty glandular parenchyma which shows homogenous signal intensity on ADC map is used as reference. The ADC values are automatically measured by drawing ROI's. The scanner software provides the mean value within the ROI which equals the ADC value [multiplied by $10(-3)]$.
The cut-off for benign and malignant lesions was set as 1.3 $\mathrm{x} 10(-3) \mathrm{mm}^{2} / \mathrm{sec}$. This was based on the study done by Savannah et al in June 2010 with 91 women.(4) In another study done by Lalithe Palle and Reddy et al in 200 patients, the sensitivity of ADC values for detection of malignant lesions was $97.2 \%$ and specificity was $100 \%$.(5)

\section{Image Interpretation}

\section{Mammogram}

Following parameters were assessed

- Glandularity and fibrofatty nature of breast parenchyma.

- Size, shape, margin, location of the lesion.

- Evidence of architectural distortion, microcalcifications suspicious of malignancy.

- Retromammary space, overlying nipple areolar complex.

- Axillary lymph node involvement.

\section{MR Mammogram}

- Glandularity of breast parenchyma.

- Lesion morphology and signal intensity in T1 and T2 weighted images were interpreted.

\section{DWI}

- Diffusion-weighted appearance of the lesion (bright or not bright).

- Absolute ADC values for each lesion were recorded.

\section{Final Diagnosis}

Histological diagnosis based on guided biopsy or resected specimen.

\section{Statistical Analysis}

- Validity parameters like sensitivity, specificity, Positive predictive value, Negative predictive value, accuracy of Diffusion-weighted imaging with histopathological diagnosis were computed.

- To test the statistical significance of the differences in the sensitivity and specificity values between the two methods, McNemar's Chi-square test was applied.

- Absolute ADC values were correlated with the histopathological diagnosis by comparing the mean ADC values between malignant and benign lesion and its statistical significance was tested by applying student's ' $\mathrm{t}$ ' test.

\section{RESULTS}

In this study, ADC cut-off for benign and malignant lesions was set as $1.3 \times 10^{(-3)}$ square $\mathrm{mm} / \mathrm{sec}$. for ADC provided $80.8 \%$ sensitivity and $100 \%$ specificity. ADC in characterising benign and malignant lesions was significant with $p$ - value of 0.004 (applying t -test). Diffusion-weighted appearances of benign and malignant lesion turned out to be statistically significant (McNemar's test, $\mathrm{p}$ value-0.18). Study also proved that BIRADS 4 categorisation on its own has a sensitivity of $84.62 \%$ and specificity of $75 \%$. 
1. Case of Fibroadenoma

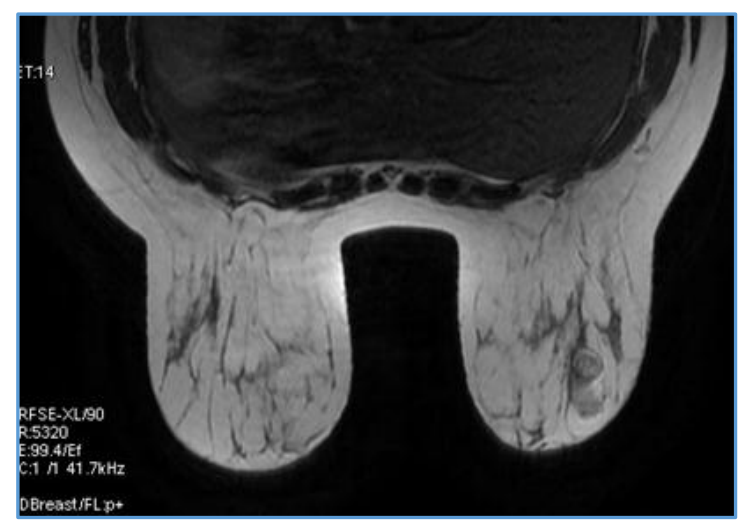

T2

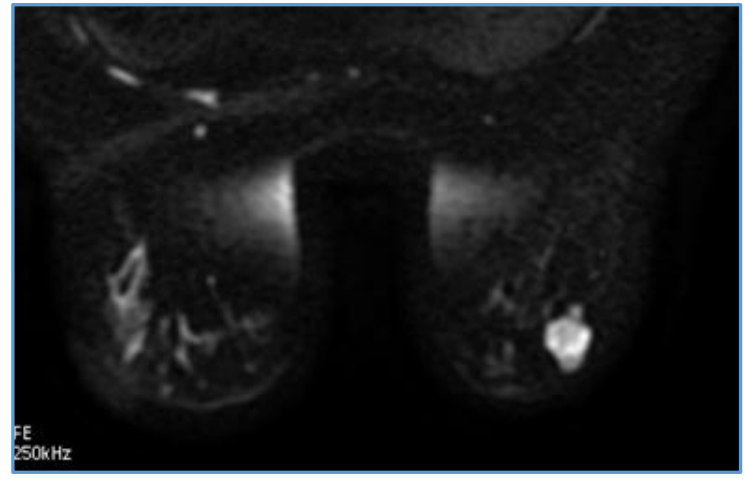

DWI

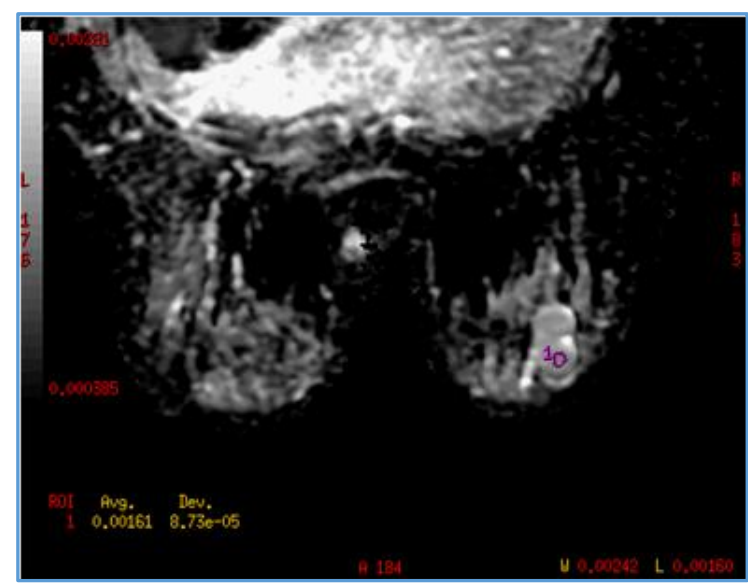

$A D C$

2. Case of Invasive Ductal Carcinoma

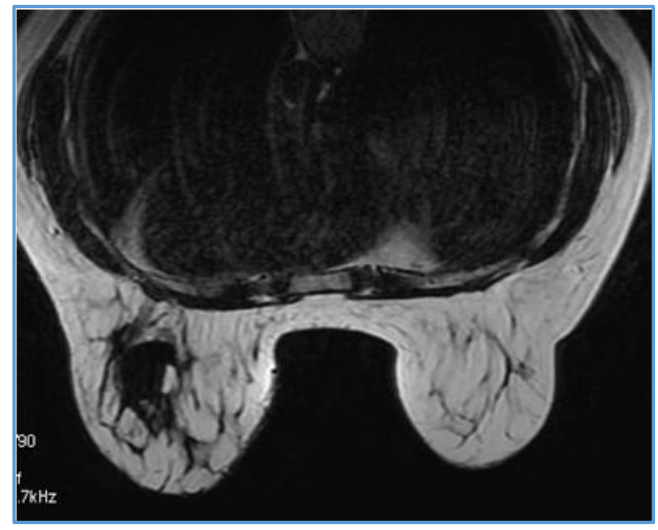

$T 2$

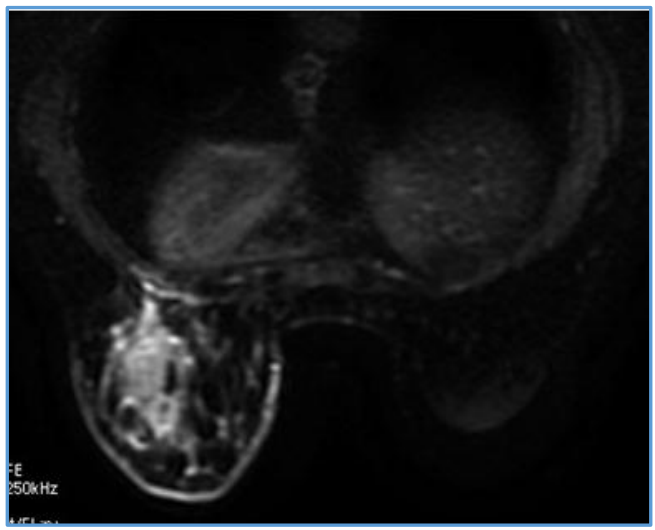

DWI

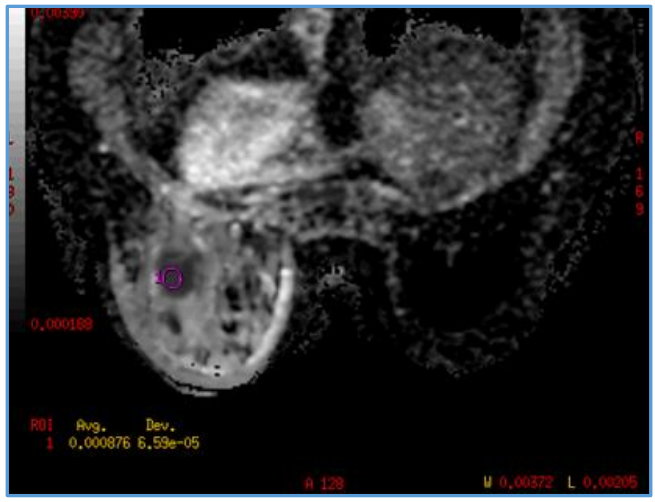

$A D C$

3. Case of Intracystic Papillary Carcinoma in Papilloma

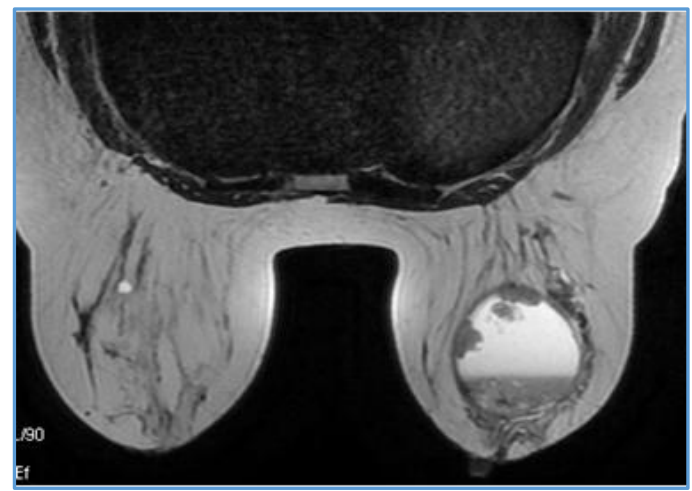

T2

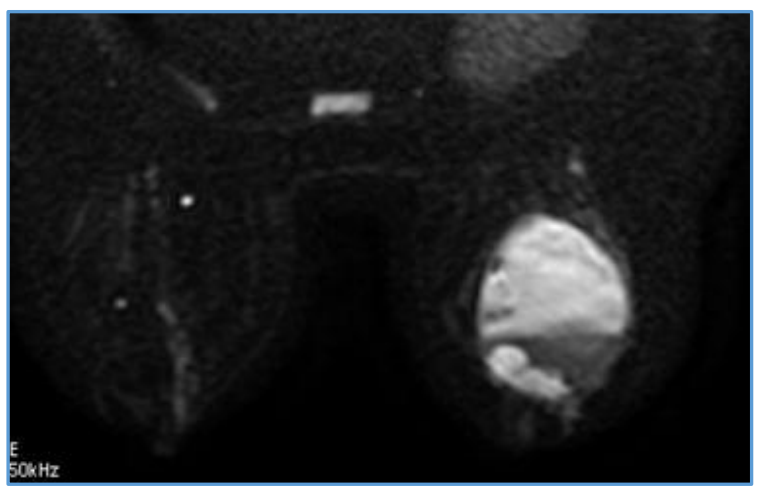

DWI 


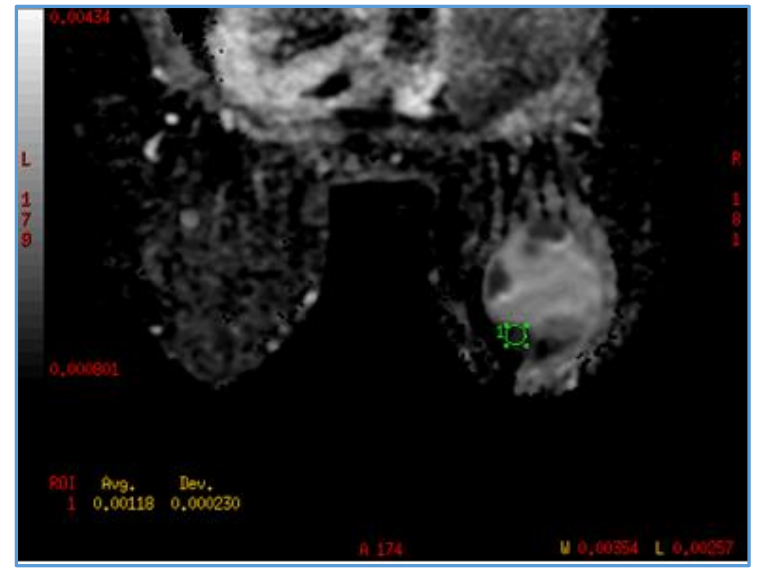

$A D C$

\section{Case of Phyllodes Tumour}

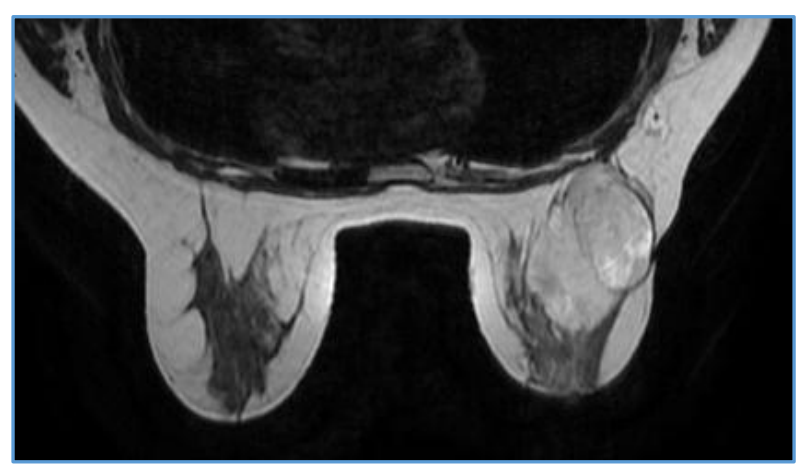

$T 2$

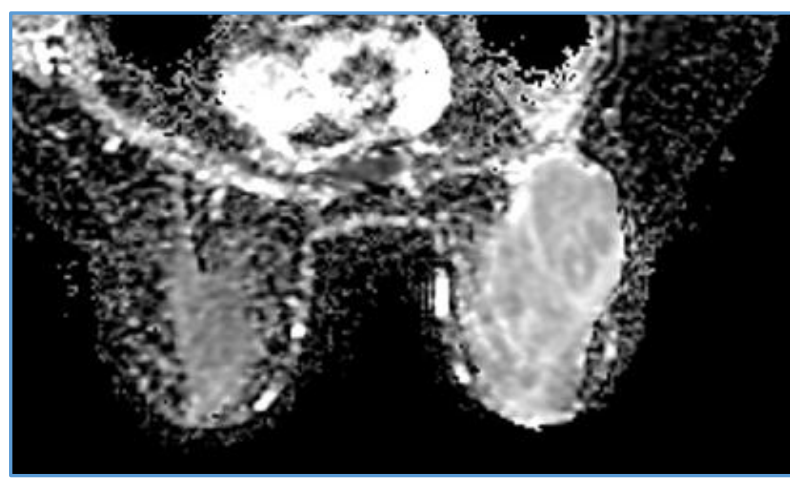

DWI

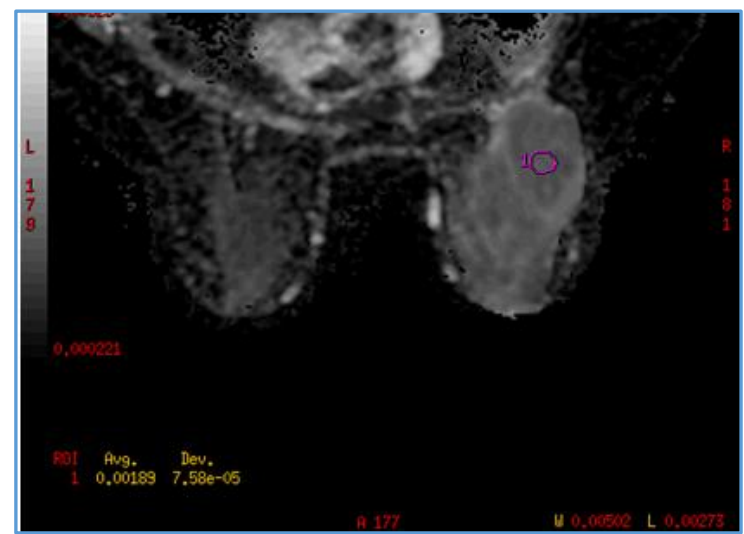

$A D C$

\section{Case of Invasive Ductal Carcinoma}

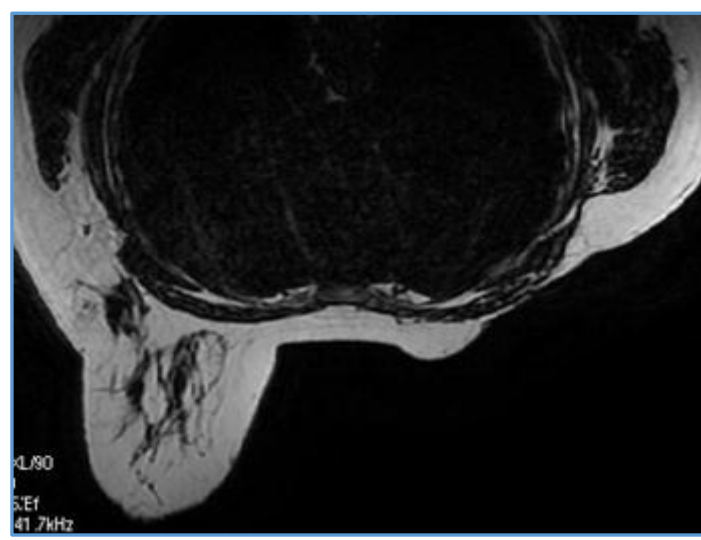

$T 2$

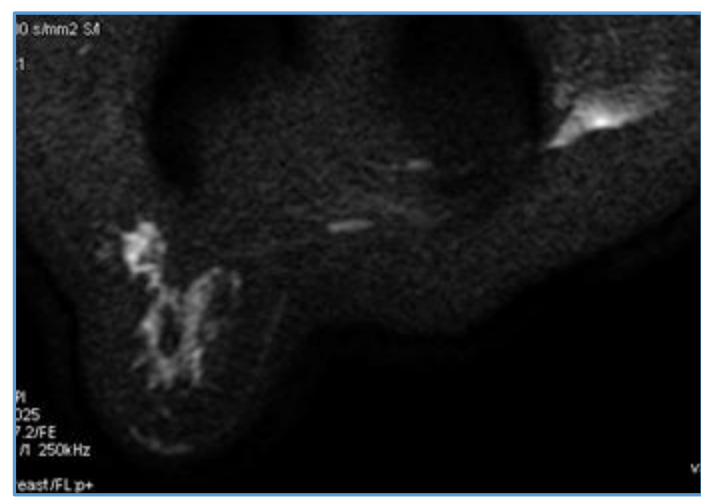

DWI

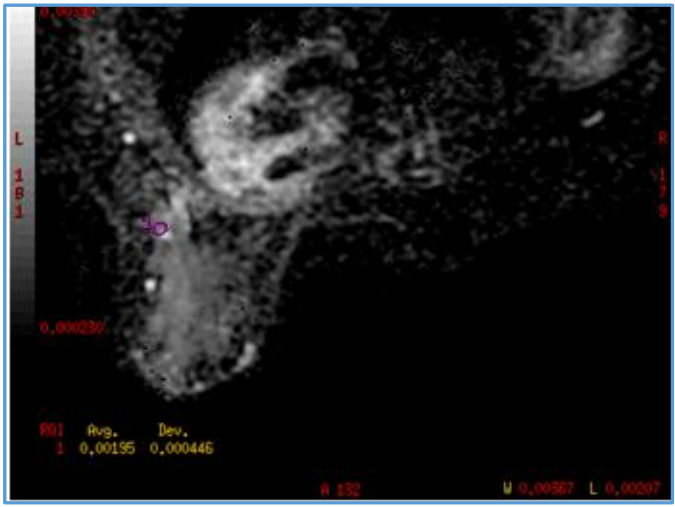

$A D C$

\section{DISCUSSION}

This study included 50 patients who are found to be in BIRADS $3 \& 4$ category on mammography. These patients underwent MRI (Axial and sagittal T1 and T2 images were obtained). DWI was performed at $b$ value of 1000 square mm/sec. ADC values were calculated by placing ROI within confines of lesion. Tissue diagnosis was obtained in all of these patients either by biopsy or resection.

Majority of the patients were in the age group between 40 60 years.

Thirty nine out of 50 women presented with a lump in the breast of which 26 were malignant. Rest of the patients presented with pain and nipple discharge. Seven patients had a previous history of malignancy of which 3 had recurrence. 
Twelve patient had dense breast of which only 3 had malignancy. In this study, there was no statistically significant association of malignancy with increasing glandularity and location of the lesion.

\section{Role of DWI and ADC in Characterising Benign and Malignant Lesion}

In this study, ADC cut-off for benign and malignant lesions was set as $1.3 \times 10(-3) \mathrm{mm}^{2} / \mathrm{sec}$. This was based on the study done by Savannah et al in June 2010 with 91 women.(4) Another study done by Lalithe Palle and Reddy et al in 200 patients, the sensitivity of this ADC cut-off for detection of malignant lesions was $97.2 \%$ and specificity was $100 \%$.(5)

Out of total 50 cases, histopathologically 24 turned out to be benign and 26 were malignant. The mean ADC for benign lesions was 1.7 and for malignant was 1.27 . This difference was statistically significant with a p-value of 0.004 . This was in concordance with the study done by Yong Guo et al in 2002 with 52 patients with a p- value of less than 0.01.(6)

Out of 26 histopathologically proved malignant lesions, 21 cases were malignant based on ADC values, giving the technique, a sensitivity of $80.8 \%$ and specificity of $100 \%$ was attained. The 5 false negative cases were small in size measuring less than $1 \mathrm{~cm}$. This inaccuracy may be due to poor resolution inherent with DWI or due to distribution of infiltrating cells, which lack cohesion and will be difficult to detect on low-spatial resolution images. This is comparable with the study done by Woodhams et al in 2011.(7)

False negative cases with higher ADC values were encountered in lesions with central necrosis and haemorrhage in a study conducted by Lalithe Palle and Reddy in 200 patients.(5) However, in our study, we did not encounter cases with central necrosis and haemorrhage.

Because of high cellularity, majority of the invasive ductal carcinomas demonstrate higher signal intensity and lower $A D C$ values than do the benign tumours.

Out of 32 diffusion bright lesions, 22 (84.6\%) turned out to be malignant and $10(41.7 \%)$ turned out to be benign with sensitivity of $84.62 \%$, specificity of $58.33 \%$, positive predictive value of $68.75 \%$ and negative predictive value of $77.78 \%$.

These false positive diffusion bright benign lesions turned out to be bloody cysts, abscess, intraductal papilloma, benign epithelial hyperplasia and fibrocystic change. This is due to high water content and high viscosity of bloody cysts and abscess. Haemorrhage may have high signal intensity and low $\mathrm{ADC}$ value, consequently it can be misdiagnosed as a malignant lesion.

Out of 18 lesions which were not bright on DWI, 14 (58.3\%) turned out to be benign and $4(15.4 \%)$ turned out to be malignant. These false negative cases were DCIS (HP Fragmented core of fibrocollagenous tissue) and Invasive ductal carcinoma with central necrosis and fibrosis. This was consistent with study conducted by Reiko Woodhams et al in 2011.(7)

Out of 40 T2 hypointense lesions, 23 (88.5\%) were malignant and 17 (70.8\%) were benign. In this study, T2 hypointensity in characterising malignant and benign lesions turned out to be statistically insignificant.
Cysts, fibroadenoma, and fibrocystic disease demonstrate high signal intensity on T2 weighted images, however, signal intensity of these benign tumours is variable on DWI and may be influenced by b-value.(7)

The combination of high signal intensity at Diffusionweighted imaging, low ADC value, and hypointensity at T2 weighted imaging is a potential indicator of malignancy. Out of 22 BIRADS 3 cases, 18 turned out to be benign (81.8\%) and 4 (18.2\%) turned out to be malignant. All the 4 malignant lesions were bright on diffusion. Out of 28 BIRADS 4 lesions, 22 (78.6 $\%)$ turned out to be malignant and $6(21.4 \%)$ turned out to be benign.

BIRADS 4 categorisation on its own had a sensitivity of $84.62 \%$ and specificity of $75 \%$.

Within the BIRADS 4 group ( $\mathrm{n}=28)$, the diffusion-weighted imaging had a sensitivity of $81 \%$ and specificity of $100 \%$ to detect malignancy. The BIRADS 4 has comparable sensitivity and specificity to ADC for detecting malignancy.

All the 4 histopathologically proved malignant BIRADS 3 lesions were bright on diffusion-weighted imaging. This shows that diffusion helps to diagnose breast cancers which may have been missed by BIRADS staging. This was consistent with retrospective study done in 171 patients by Mitsuhiro et al in 2009.

The results suggest that DWI may be helpful in reducing the number of unnecessary biopsies following categorisation into BIRADS 3 and 4 group. ADC values are useful in differentiating between DCIS and invasive breast carcinoma as well as in prognostication in a study done by S Y Chovi et al.(8)

A ROC curve analysis was used to determine the optimum cut-off values of mean ADC among malignant and benign lesions. Accordingly, the cut-off values of ADC $1.25 \times 10(-3)$ $\mathrm{mm}^{2} / \mathrm{sec}$ was derived with $80.8 \%$ sensitivity and $100 \%$ specificity and area under the curve (AUC -0.841) for ADC was obtained to differentiate malignant from benign diseases.

Diffusion-weighted imaging is a promising new tool for the characterisation of suspected breast cancer. When used in conjunction with mammography and conventional MRI, diffusion-weighted imaging has a potential to diagnose malignancy which would be otherwise missed. A study with a larger cohort of patients with more number of DCIS patients would further clarify the role of DWI in carcinoma breast.

\section{Draw Backs and Limitations}

- Voluntary patient movement, a potential source of image artifact by causing image misregistration, which leads to false ADC values.

- Low spatial resolution- Small cancer foci including DCIS and scattered foci of invasive lobular cancer may not be depicted at DWI. High SNR and high b-value can be used to increase the spatial resolution of DWI thereby allowing detection and characterisation of smaller lesions.

- Haemorrhage may have high signal intensity and low ADC values, misdiagnosed as malignant lesions. Fat suppressed T1 weighted images need to be interpreted in conjunction with DWI to avoid misdiagnosis.

- Small-sized lesions could not be researched thoroughly. Study with different ranges of "b" values could not be conducted due to time constraints. ${ }^{(7)}$ 


\section{CONCLUSION}

- $\quad$ ADC is an effective and precise parameter to identify and distinguish between malignant and benign breast lesions. The corresponding ADC values which can be used to distinguish benign and malignant lesions are also derived as a part of the study. The cut-off values of ADC $1.25 \mathrm{X} 10$ (3) $\mathrm{mm} 2 / \mathrm{sec}$ was derived with $80.8 \%$ sensitivity and $100 \%$ specificity.

- Diffusion-weighted imaging is found to be useful in diagnosis of BIRADS 3 and 4 lesions.

- ADC values reported among studies vary widely due to widespread difference in MR protocols. Therefore, standardisation of diffusion-weighted protocols need to be established to ensure reproducibility at various centres. This will contribute to the implementation of ADC measurement for tumour characterisation in future.(9)

\section{REFERENCES}

[1] Lee SC, Jain PA, Jethwa SC, et al. Radiologists' role in breast cancer staging: providing key information for clinicians. Radiographics 2014;34(2):330-42.

[2] Rajeshkannan R, Moorthy S, Sreekumar KP, et al. Clinical application of diffusion weighted MR imaging: a review. Ind J RadiolImag 2006;16(4):705-10.

[3] Kul S, Cansu A, Alhan E, et al. Contribution of Diffusionweighted imaging to dynamic contrast-enhanced MRI in the characterization of breast tumours. AJR 2011;196(1):210-7.
[4] Partridge SC, DeMartini WB, Kurland BF, et al. Quantitative Diffusion-weighted imaging as an adjuvant to conventional breast MRI for improved positive predictive value. AJR 2009;193(6):1716-22.

[5] Palle L, Reddy B. Role of diffusion MRI in characterizing benign and malignant breast lesions. Indian Journal of Radiology and Imaging 2009;19(4):287-90.

[6] Guo Y, Cai YQ, Cai ZL, et al. Differentiation of clinically benign and malignant breast lesions using Diffusionweighted imaging. J Magn Reson Imaging 2002;16(2):172-8.

[7] Woodhams R, Ramadan S, Stanwell P, et al. Diffusionweighted imaging of the breast: principles and clinical applications. Radiographics 2011;31(4):1059-84.

[8] Choi SY, Chang YW, Park HJ, et al. Correlation of the apparent diffusion coefficiency values on Diffusionweighted imaging with prognostic factors for breast cancer. Br J Radiol 2012;85(1016):e474-9.

[9] Vermoolen MA, Kwee TC, Nievelstein RAJ. Apparent diffusion coefficient measurements in the differentiation between benign and malignant lesions: a systematic review. Insights imaging 2012;3(4):395-409. 